\title{
Functional Connectivity Dynamics Among Cortical Neurons: A Dependence Analysis
}

\author{
Lin Li, Student Member, IEEE, Il Memming Park, Sohan Seth, Student Member, IEEE, \\ Justin C. Sanchez, Member, IEEE, and José C. Príncipe, Fellow, IEEE
}

\begin{abstract}
This paper quantifies and comparatively validates functional connectivity between neurons by measuring the statistical dependence between their firing rates. Based on statistical analysis of the pairwise functional connectivity, we estimate, exclusively from neural data, the neural assembly functional connectivity given a behavior task, which provides a quantifiable representation of the dynamic nature during the behavioral task. Because of the time scale of behavior $(100-1000 \mathrm{~ms})$, a statistical method that yields robust estimators for this small sample size is desirable. In this work, the temporal resolutions of four estimators of functional connectivity are compared on both simulated data and real neural ensemble recordings. The comparison highlights how the properties and assumptions of statistical-based and phase-based metrics affect the interpretation of connectivity. Simulation results show that mean square contingency (MSC) and mutual information (MI) create more robust quantification of functional connectivity under identical conditions than cross correlation (CC) and phase synchronization $(\mathrm{PhS})$ when the sample size is $1 \mathrm{~s}$. The results of the simulated analysis are extended to real neuronal recordings to assess the functional connectivity in monkey's cortex corresponding to three movement states in a food reaching task and construct the assembly graph given a movement state and the activation degree of a state-related assembly over time using the statistical test exclusively from neural data dependencies. The activation degree of a given state-related assembly reaches the peak repeatedly when the specific movement states occur, which also reveals the network of interactions among the neurons are key for the operation of a specific behavior.
\end{abstract}

Index Terms-Dependence measure, functional connectivity dynamics.

\section{INTRODUCTION}

$\mathbf{T}$ HE NEURAL mechanisms that select and coordinate the distributed cortical activity to produce movement have been the focus of system neuroscience [1]. However, there are significant challenges to quantify their nature. The development

Manuscript received December 31, 2010; revised July 25, 2011; accepted October 30, 2011. Date of publication December 20, 2011; date of current version January 25, 2012. This work was supported in part by the U.S. National Science Foundation under Grant CNS-0540304, in part by NSF Partnerships for Innovation Program 0650161, and in part by DARPA project N66001-10-C-2008.

$\mathrm{L}$. Li and S. Seth are with the Department of Electrical Engineering, University of Florida, Gainesville, FL 32611 USA (e-mail: linli@ cnel.ufl.edu; sohan@cnel.ufl.edu).

I. M. Park is with Neural Coding and Computation Lab, University of Texas at Austin, Austin, TX 78712 USA (e-mail: memming@gmail.com).

J. C. Sanchez is with the Department of Biomedical Engineering, University of Miami, Coral Gables, FL 33146 USA (e-mail: jcsanchez@miami.edu).

J. C. Príncipe is with the Department of Electrical Engineering and Biomedical Engineering, University of Florida, Gainesville, FL 32611 USA (e-mail: principe@cnel.ufl.edu).

Color versions of one or more of the figures in this paper are available online at http://ieeexplore.ieee.org.

Digital Object Identifier 10.1109/TNSRE.2011.2176749 of new tools to assess this activation is critical because neural assemblies provide a conceptual framework for the integration of distributed and individual neural firings. The assemblies are defined as distributed local networks of neurons transiently linked by active dynamic couplings [2]. It is generally believed that the emergence of specific neuronal assemblies underly cognitive actions [1], [3]. Due to the spatially specific and transient nature of the inter neuron communication, quantification of such neural assemblies is a difficult task.

In motor brain-machine interfaces (BMIs), the goal is to decode the intention of movement, using multielectrode neural data from motor cortex synchronized with kinematic variables measurements. From a statistical modeling perspective, the complete information to solve this problem resides on the joint probability density function of the multivariate neural data and of the multivariate kinematic variables, but this is never done due to the high dimension of the joint distributions. Even when we develop models that estimate the conditional probability of the kinematics given the neural data, we do not use the joint information expressed in the multichannel neural data for the same reason. Instead, we model the conditional dependence only with respect to the history of a vector of neural spike trains. Since each neural channel is nothing but the marginal density of the joint probability of multivariate neural data, this procedure is only a reasonable solution when the neurons spike independently. In this study, we aim to quantify and analyze pairwise dependencies between cortical neurons involved in a given behavioral task using the information from neural data conditioned on the kinematic information. In this paper the dependencies that occur transiently in time between pairs of neurons will be named interactions, and their spatiotemporal patterns are interpreted as signatures of functional connectivity. Our hypothesis is that given a kinematic state defined by the experiment, specific neural assemblies are transiently activated, which can be quantified by a significant enhancement of the dependencies between pairs of neurons, i.e., their functional connectivity.

The functional connectivity is commonly quantified by correlation, coherence, and synchrony [4]-[8]. Correlation and coherence capture linear relations in time and frequency domain respectively, while (statistical) synchrony measures the simultaneous firing of neurons in excess of coincidental firings. However, in general, the interaction between neurons can be highly nonlinear and not exclusively in the form of synchrony [9]. The general form of interaction can be quantified by statistical means, namely by dependence measures, which estimate arbitrary relationships between two random variables. For examples see [10]-[12]. 
Neural assemblies have a transient, dynamic existence that spans the time scale of behavior. The activation of a neural assembly is long enough for neural activity to propagate through the assembly. A propagation that necessarily involves cycles of reciprocal spike exchanges with transmission delays lasts tens of milliseconds. In order to quantify and study this interaction during movements, we need to focus on the temporal dynamics of neural networks in the hundreds to thousands of millisecond range. Due to the sparse firing of cortical neurons, in this paper we first concentrate on comparing statistical methods that support small sample estimators of dependence. Four methods, mean square contingence (MSC), mutual information (MI), phase synchronization $(\mathrm{PhS})$, and cross correlation $(\mathrm{CC})$ are tested on synthetic spike trains generated by a network of leaky integrate-and-fire neurons [13]. We select two baseline methods with different properties: $\mathrm{CC}$ measures the linear relation between two neuron activities in time, while $\mathrm{PhS}$ measures the pairwise synchrony of neurons. In contrast, MI and MSC quantify nonlinear dependence of two nominal variables based on two major independence tests: Chi-square test and G-test of independence. MI and MSC calculate the Kullback-Leibler divergence and $\chi^{2}$ divergence of the joint probability distribution with respect to the product of the two marginal probability distributions, respectively. Although both MI and MSC are widely used in applied statistics measurements, their capability in estimating functional connectivity with small sample size has not been fully investigated. See [14] for the use of MI in neural assemblies. We use statistical power and dispersion criteria to evaluate the performance of these four methods as a function of the sample size. Because of the robustness with small window size, MSC is selected as the estimator to analyze the dynamic functional connectivity associated with the movement state.

Furthermore, the dependence measure is applied to monkey cortical neural activity recorded during a food reaching task with three well defined kinematic states. The estimated functional connectivity describes the assemblies associated with the time varying kinematic states. The nodes (neurons) and edges (the functional connections between pairs of neurons) in an assembly graph represent different aspects of the time spatial neuronal interactions. Specifically, we visualize the local assembly graph within four cortical areas during reaching tasks.

We illustrate the correlation between the evolution of the connection strength and the transition from the rest to movement state. This correlation was also established in the past using a supervised framework (i.e., with the knowledge of the desired response [15] or using a hidden Markov model trained with pre segmented data [16]), however here it is decided with a statistical test exclusively from neural data dependencies.

Moreover, we estimate the activation degree of a specific assembly based on the number of activated edges in the graph. The activation degree of a state-related assembly reaches a peak when the corresponding kinematic state begins, which also reveals that the network of interactions among the neurons is the key factor for the operation of a specific behavior. The evolution of this complex local functional connectivity over the cortical space and over time can be readily visualized in a movie ${ }^{1}$ or in static graphs as we demonstrate in Section V.

${ }^{1}$ http://cnel.ufl.edu/research/linli.php
The rest of the paper is organized as follows. Section II introduces different dependence measures of functional connectivity between a pair of neuron-firing-rate time series. Section III discusses the simulated neural circuit for the method performance comparison. The performance comparison criteria are introduced in Section IV. The comparison results are shown in Section V. The monkey cortical neural data during a food reaching experiment are described in Section VI. In Section VII, the selected dependence measure are applied on monkey data to estimate the kinematic-state-related assembly and investigate the association between the functional connectivity dynamics and the kinematic state. Section VIII visualizes the dynamic local functional connectivity of each cortical area and analyzes its correlation with the regional contribution to the movement implementation, and Section IX concludes this paper.

\section{Functional Connectivity Measures}

The existence of massive anatomical connections among cortical neurons does not make them functionally connected all the time. Even in the absence of neural code knowledge, exchange of information across the neuropil can be spotted and quantified externally by the co-occurrence in time of action potentials produced by neurons. In this paper, we propose to quantify functional connectivity by the statistical dependence between neural firing rates. But first we briefly review different neural dependence measures. Consider two discrete firing rate time series $x_{n}$ and $y_{n}, n=1, \ldots, N$, recorded simultaneously and produced by two neurons. Each dependence measure has particular characteristics and we divide them into two groups: statistical-based and phase-based.

\section{A. Statistical Measures}

1) Second-Order Measures: The most widely used technique to measure the similarity between two time series of firing rate $x$ and $y$ is the cross-correlation, defined in the time domain as a function of the time lag $\tau=-(N-1), \ldots, 0, \ldots, N-1$

$$
C_{Y X}(\tau)\left\{\begin{array}{ll}
=\frac{1}{N-\tau} \sum_{n=1}^{N-\tau}\left(\frac{x_{n+\tau}-\bar{x}}{\sigma_{x}}\right)\left(\frac{y_{n}-\bar{y}}{\sigma_{y}}\right) & \tau \geq 0 \\
=C_{Y X}(-\tau) & \tau \leq 0
\end{array} .\right.
$$

The cross correlation is obtained by normalizing the cross covariance and thus ranges from minus one (anti-phase correlation) to one (in-phase correlation), while values close to zero are attained for uncorrelated time series. In time series analysis using a linear generative model with Gaussian inputs, the cross correlation coefficient quantifies totally the dependence between the time series.

As a measure of functional connection between two neurons, the cross correlation coefficient is calculated between two firing rate time series with a certain bin size. In this study we selected the bin size at $100 \mathrm{~ms}$, not because it is the best choice but because it is a reasonable compromise for the application. From multiscale studies on spike trains for BMIs [17] and also from the analysis of function connectivity in neural ensembles [18] it is known that the best approach to functional connectivity is to use multiple time scales to analyze spike trains. However, this 
methodology is rather expensive computationally and it is incompatible with online studies as the ones we envisage in this work. Therefore, we have to select a single bin size that is a good compromise between specificity of neural firing and sufficient similarity with behavior. From [17] $100 \mathrm{~ms}$ is the time scale that bridges the gap between reasonable correlations with the movements and a reasonable specificity regarding neural firings. Moreover, since the average firing rate of cortical neurons is around $10 \mathrm{~Hz}$, with $100 \mathrm{~ms}$ bin size, the average spike number per bin is 1 , which yields a suitable sparseness for estimating dependency. Because the neural data we used is collected from neighboring cortical regions and we quantify firing rates with bin size of $100 \mathrm{~ms}$, we can expect that the dependence induced by the functional connection between a pair of neurons usually happens within the $100 \mathrm{~ms} \mathrm{lag}$. Therefore, only the correlation with lag zero is calculated. However, cross correlation only indicates the strength of a linear dependence between two variables, but its value cannot completely characterize their relationship that may appear in higher order statistical moments (skewness, kurtosis, etc.).

2) Dependence Measures: In contrast to cross-correlation, both MI and MSC quantify the statistical dependencies between two random variables using the full statistical information in their joint space, with no assumption about the form of their respective densities and implicitly their generating processes, which can be linear or nonlinear. It is therefore more appropriate when the data generation mechanism is not fully understood, but it is much more difficult to estimate in practice.

3) Mean Square Contingency: Mean Square Contingency (MSC) is a measure of dependence based on an independence test, and it was first defined by Pearson for two discrete random variables [19]. MSC calculates the normalized $\chi^{2}$ divergence between the joint probability distribution and the product of the two marginal probability distributions of two firing-rate time series [10].

For a test of independence, an observation consists of a pair of values and the null hypothesis is that the occurrences of these values are statistically independent. Each observed probability is allocated to one cell of a two-dimensional array (known as contingency table) according to its values. If there are $r$ rows and $c$ columns in the table, the theoretical probability for a cell given the independent assumption is $E_{i, j}=P_{i+} P_{+j}, P_{i+}=$ $\sum_{k=1}^{c} P_{i, k}$ and $P_{+j}=\sum_{k=1}^{r} P_{k, j}$ are the marginal probability, and $P_{i, j}$ is the observed (joint) probability of the cell in row $i$ and column $j$ in contingency table.

The MSC value, also called the Pearson contingency coefficient, denoted by $P$, is given by

$$
P=\left(\frac{\Phi^{2}}{1+\Phi^{2}}\right)^{1 / 2}
$$

where

$$
\begin{aligned}
\Phi^{2} & =\sum_{i=1}^{r} \sum_{j=1}^{c} \frac{\left(P_{i, j}-E_{i, j}\right)^{2}}{E_{i, j}} \\
& =\sum_{i=1}^{r} \sum_{j=1}^{c} \frac{P_{i, j}^{2}}{P_{i+} P_{+j}}-1 .
\end{aligned}
$$

Note that this statistic basically scales the $\chi^{2}$ statistic to a value between 0 (independence) and 1 (maximum dependence). It has the desirable property of scale invariance. That is, the value of Pearson's contingency coefficient does not change as long as the probabilities remain constant. Unfortunately, the estimation is biased when the sample size is small. William's correction is used to decrease the inaccuracy [20] and the bias corrected expression $P^{\infty}$ is

$$
P^{\infty}=\left(\frac{\Phi^{2}}{q+\Phi^{2}}\right)^{1 / 2}
$$

where

$$
q=1+\frac{\sum_{i=1}^{r} \frac{1}{P_{i+}} \sum_{j=1}^{c} \frac{1}{P_{+j}}}{6 N(r-1)(c-1)}
$$

where $N$ is the total sample size.

4) Mutual Information: In contrast with MSC, MI quantifies the KL-divergence between the joint probability distribution and the product of the two marginal probability distributions, which is used to determine the synaptic connection structure of neuronal networks [21]. According to the contingency table, the Shannon entropy of the row $(\mathrm{X})$ and column $(\mathrm{Y})$ vector are defined, respectively, as

$$
\hat{H}(X)=-\sum_{i=1}^{r} P_{i+} \ln P_{i+}, \hat{H}(Y)=-\sum_{i=1}^{c} P_{+j} \ln P_{+j}
$$

where $P_{i+}$ and $P_{+j}$ are the marginal probability and $P_{i j}$ is the observed (joint) probability of the cell in row $i$ and column $j$ in contingency table. The estimation of $H$ (row) and $H$ (column) is heavily biased negatively when the contingency table contains zero entries $\left(P_{+j}=0\right.$ or $\left.P_{i+}=0\right)$ although the true probability is nonzero, which is likely to occur with a small sample size compared to the number of entries of the table. The first-order bias corrected entropy $H^{\infty}$ [22], is then defined as

$$
\begin{aligned}
H^{\infty}(X) & =\hat{H}(X)+\frac{b_{X}-1}{2 N} \\
H^{\infty}(Y) & =\hat{H}(Y)+\frac{b_{Y}-1}{2 N}
\end{aligned}
$$

where $b_{X}$ and $b_{Y}$ are the number of states for which $P_{i+} \neq 0$ and $P_{+j} \neq 0$, respectively. The joint entropy between $X$ and $Y$ is defined as

$$
\hat{H}(X Y)=-\sum_{i, j} P_{i, j} \ln P_{i, j} .
$$

The mutual information between $X$ and $Y$ is defined as

$$
\hat{M} I(X, Y)=\hat{H}(X)+\hat{H}(Y)-\hat{H}(X, Y)
$$

and its bias corrected expression $M I^{\infty}$ is

$$
M I^{\infty}(X, Y)=\hat{M} I(X, Y)+\frac{b_{X}+b_{Y}-b_{X Y}-1}{2 N}
$$

where $b_{X Y}$ are the number of cells in contingency table greater than zero. In the following, the mutual information is bias corrected and also normalized by dividing it by $\ln (N)$. 


\section{B. Phase Synchronization Measures}

Phase synchronization $(\mathrm{PhS})$ is an ubiquitous phenomenon in many physical oscillatory systems, which also occurs in neural structures. However, the methodology is based on deterministic dynamical system principles that may not apply to neural structures due to their intrinsic stochastic nature. Nevertheless, phase synchronization has been widely applied to neural systems [23]. In this paper, we apply $\mathrm{PhS}$ to the time series of firing rate. We view the time series of firing rate as a continuous signal $x(t)=x_{n}, n \Delta \leq t<(n+1) \Delta$, where $\Delta=100$ ms represents the bin size. This long bin width is sufficiently long to smooth short term fluctuations produced by neural stochasticity. Phase synchronization indicates the following phase locking condition applied for any time $t, \varphi(t)=\left|n \phi_{x}(t)-m \phi_{y}(t)\right| \leq$ constant, where $\phi_{x}(t)$ and $\phi_{y}(t)$ represent the phase of the signal recorded from the neuron $\mathrm{x}$ and $\mathrm{y}$, respectively [23]. In the following computations we take the terms with $|m|=|n|=1$. Basically, phase synchronization analysis proceeds into two steps: 1) estimation of the instantaneous phases and 2) quantification of the phase locking.

1) Estimation of Instantaneous Phases: In this study, the phase is extracted via Hilbert transform, although there are many other methods [24] (for a survey of methods, we refer to [23]). The analytic signal $\xi(t)$ of the univariate measurement is a complex function of continuous time $x(t)$ defined as

$$
\xi(t)=x(t)+i x_{h}(t)=a_{\xi}(t) e^{j \phi_{\xi}(t)}
$$

where the function $x_{h}(t)$ is the Hilbert transform of $x(t)$

$$
x_{h}(t)=\frac{1}{\pi} \mathbf{P} \cdot \mathbf{V} \cdot \int_{-\infty}^{+\infty} \frac{x(t)}{t-\tau} d \tau .
$$

$\mathbf{P . V}$. indicates that the integral is taken in the sense of Cauchy principal value. $a_{\xi}(t)$ and $\phi_{\xi}(t)$ are the instantaneous amplitude and phase of the analytic signal $\xi(t)$ of $x(t)$. The instantaneous phase $\phi_{x}(t)$ is taken equal to $\phi_{\xi}(t)$, while the phase $\phi_{y}(t)$ is estimated from $y(t)$ following the same procedure for $\operatorname{phi}(\mathrm{x})$.

2) Phase-Locking Quantification: For neural signals, the phase locking condition can be better understood in a statistical sense, by estimating in the distribution of cyclic relative phase $\varphi^{\prime}(t)=\varphi(t) \bmod 2 \pi$ [25]. For independent time series $x(t)$ and $y(t)$, the distribution of the relative phase $\varphi^{\prime}(t)$ is uniform within a given time window. The detection of phase locking involves quantifying how far from uniform is the relative phase distribution. Several measures have been proposed for this purpose [26], [27]. The one we use is [26]

$$
\rho=\left\|\frac{1}{N} \sum_{j=1}^{N} e^{i \varphi^{\prime}\left(t_{j}\right)}\right\|
$$

where $N$ is the number of sample in a window and $\rho$ is the mean phase coherence of the angular distribution [28]. By construction, it is bounded by 0 (no synchronization) and 1 (perfect synchronization).

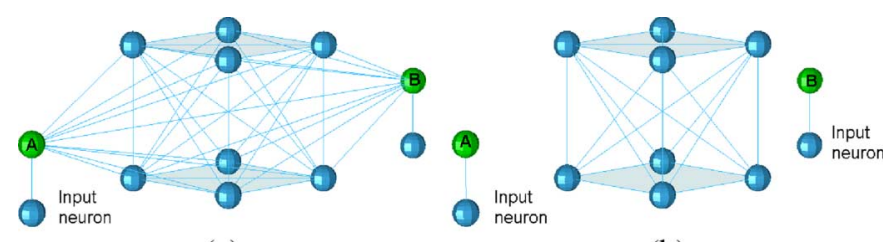

(a)

(b)

Fig. 1. The stimulated network of 10 neurons. Neurons A and B are selected from the cell assembly for measurement. Neuron A and neuron B are excited by independent spike trains. The spike trains of neuron $\mathrm{A}$ and $\mathrm{B}$ are recorded in DIS and CON state for the interaction analysis. (a) CON state (the cell assembly is fully connected). (b) DIS state (the cell assembly is disconnected (direct and indirect) between neuron $\mathrm{A}$ and $\mathrm{B}$ ).

\section{Simulated DATA}

We test the capability of the four methods in estimating functional connectivity as a function of the window size of observations. The experiment is performed in a neural Circuit SIMulator (CSIM) [13], using a network of 10 neurons organized as a cell assembly. Given the parameters of the simulation, this number is sufficient to generalize the conclusions of their performance for larger network sizes [29]. Two neurons, A and B from the cell assembly, are selected for measurement. The spike trains are recorded in two states: the cell assembly is either fully connected [CON state as shown in Fig. 1(a)] or disconnected (direct and indirect) between neuron A and B [DIS state as shown in Fig. 1(b)]. The interaction between neuron A and B is estimated based on dependence measures.

All neurons are modeled as leaky integrate-and-fire (LIF) units, since the integrate-and-fire neuron model is one of the most widely used spiking neuron models for analyzing the behavior of neural systems. It provides practical and relatively realistic descriptions of the neuron membrane potential (spike) in terms of the synaptic inputs and the injected current that it receives. Neuron parameters are [30]: membrane time constant $30 \mathrm{~ms}$, absolute refractory period $3 \mathrm{~ms}$ (excitatory neurons), threshold $15 \mathrm{mV}$ (for a resting membrane potential assumed to be 0 ), reset voltage $14.3 \mathrm{mV}$, constant nonspecific background current $13.5 \mathrm{nA}$, input resistance $1 \mathrm{M} \Omega$, and input noise $9 \mathrm{nA}$. The postsynaptic current is modeled as an exponential decay $W \exp \left(-t / \tau_{s}\right)$ with $\tau_{s}=3 \mathrm{~ms}$. The average synaptic weight $W$ is 1 . All neurons fire at a reasonable firing rate range (1-10 Hz), as shown in Fig. 2. For analysis, neuronal spike events are binned in nonoverlapping windows of $100 \mathrm{~ms}$.

\section{CRITERION FOR COMPARING DiFFERENT MEASURES}

To compare the different measures of functional connectivity in terms of their time resolution, the statistical power analysis and the variance analysis are utilized to evaluate their capability to detect connectivity and the precision of the connection strength estimation, respectively.

Statistical power estimates the probability of detecting a functional connection given that a functional connection exists with a fixed type-I error [31]. We assume DIS state and CON state to be the null-hypothesis and the alternative-hypothesis, respectively. Since the mean value of dependence in CON state is larger than that in DIS state, we perform a one-tail hypothesis test. The threshold is defined by the dependence of DIS 

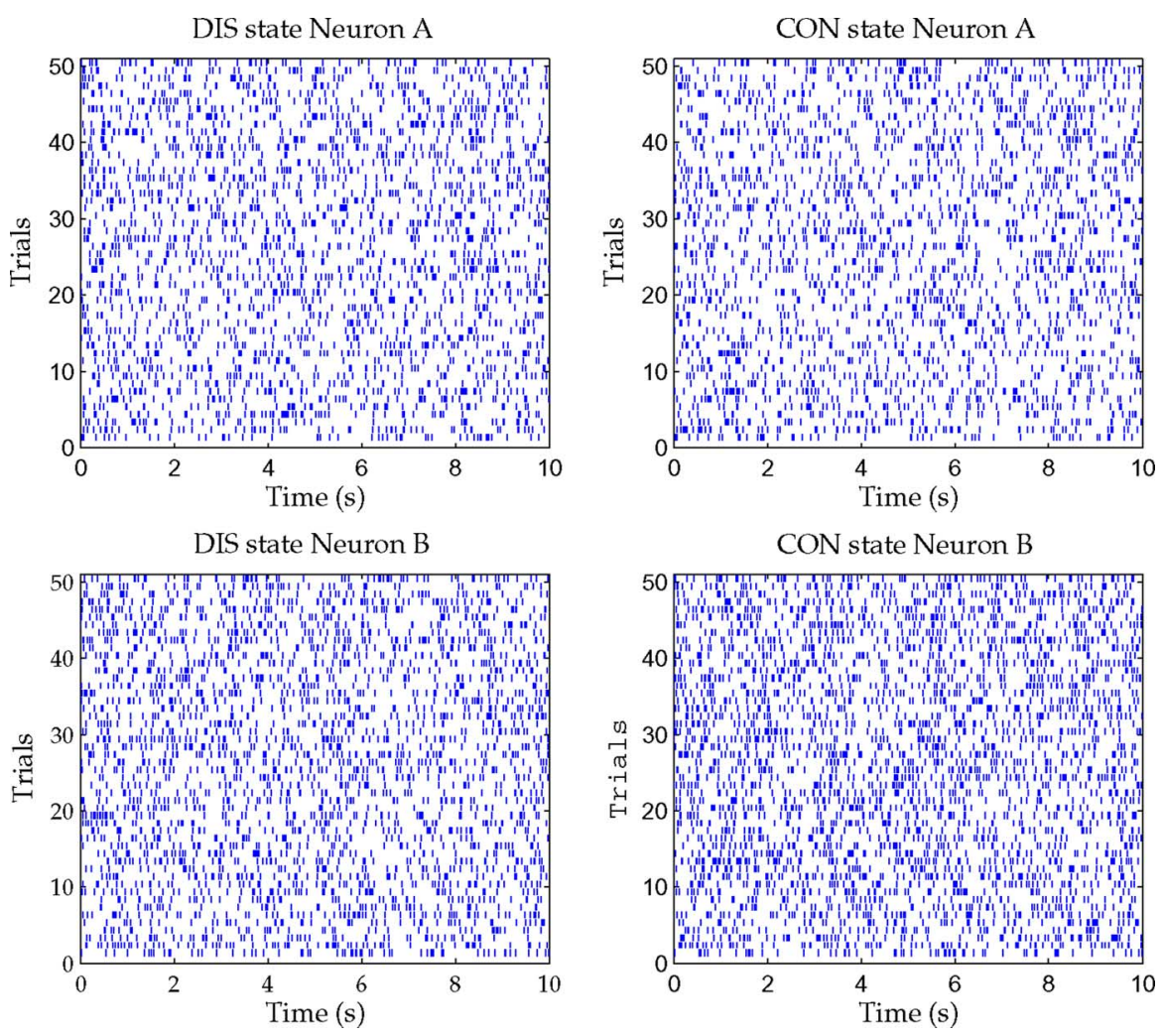

Fig. 2. The raster plots of neuron A and B under DIS and CON state. Under each state, neurons A and B fire at a reasonable firing rate range (1-10 Hz).

state with the significance of 0.05 . The statistical power is the probability that the dependence of CON state is larger than the threshold.

We compare the performance of four methods as a function of window sizes by computing the statistical powers. With the same window size, the larger statistical power indicates the better capability of detecting functional connectivity.

In addition, we also consider the dependence variance as a criterion. Because the dependence value is used to describe the connection strength, the method with a smaller variance is preferred as a more precise connectivity estimator.

\section{Simulation Results}

More samples usually enable the measure of functional connectivity with a better accuracy. However, because the time scale of behavior is so short, $100-1000 \mathrm{~ms}$, a statistical method that yields a robust estimator for 10 samples is desired. To compare the performance among different statistical estimators with respect to the window size, we vary the length of the time window applied to the signal from 1 to $10 \mathrm{~s}$ (nonoverlapping windows with 10-100 samples collected with a period of $100 \mathrm{~ms})$.

We run the simulation in 100 random settings of the synaptic weights between pair of neurons based on uniform distributed realization of the truncated normal distribution $W \sim N(1,0.1)$, $W \in[0,2]$. In each setting, the simulation generates 200 subsets of neural activity in DIS state and CON state, respectively. The dependence is measured for each subset with different window sizes. The statistical power of dependence is calculated for each random setting with respect to the window size. Fig. 3(a) shows that the statistical power of the coupling detection is a monotonically increasing function of window size. The phase synchronization increases the most, which suggests that phase synchronization is progressively more efficient with longer time windows that characterize stable regimes. In contrast, the mean square contingency and MI have a more gradual increase indicating that their performance is the least sensitive to the window length. The statistical power of MI is the best over all window sizes, while the statistical power for cross-correlation is always lower than that of other dependence estimators, because the nonlinear coupling is not detected well by cross-correlation.

Moreover, a Kolmogorov-Smirnov Test (KS-test) is performed on statistical powers of each pair of measures, which indicates that the statistical power of MI and MSC is significantly greater than that of cross-correlation and phase synchronization when the window size is small, e.g., 10 and 20 samples. With 10-sample window size, when we decrease the estimation difficulty by increasing the average synaptic weight $\mathrm{W}$, the statistical power of MI and MSC is significantly greater than that of cross-correlation and phase synchronization, as shown in Table I. This reflects the robustness of MI and MSC with small window estimation. This robustness suggests that MI and MSC are better candidates to estimate the dynamic functional connectivity.

To further investigate each measure's capability for small window estimation, we present the variance of the estimated connection based on four methods in Fig. 3(b). The standard deviation decreases with increasing window size, as it can be expected. However, if one is interested in small window sizes to quantify the fast dynamics of neural assemblies, we 


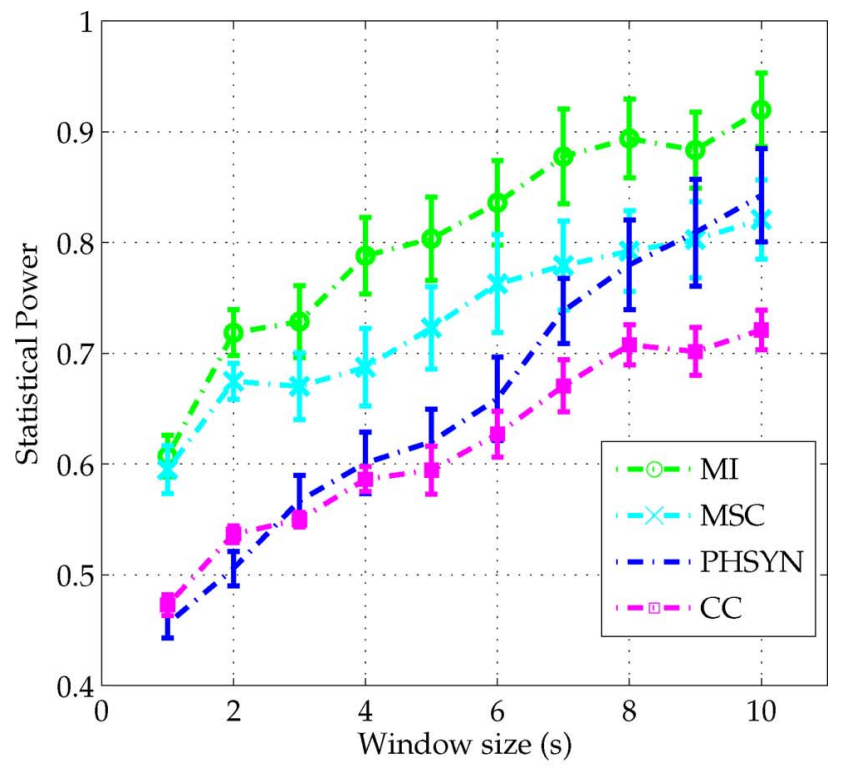

(a)
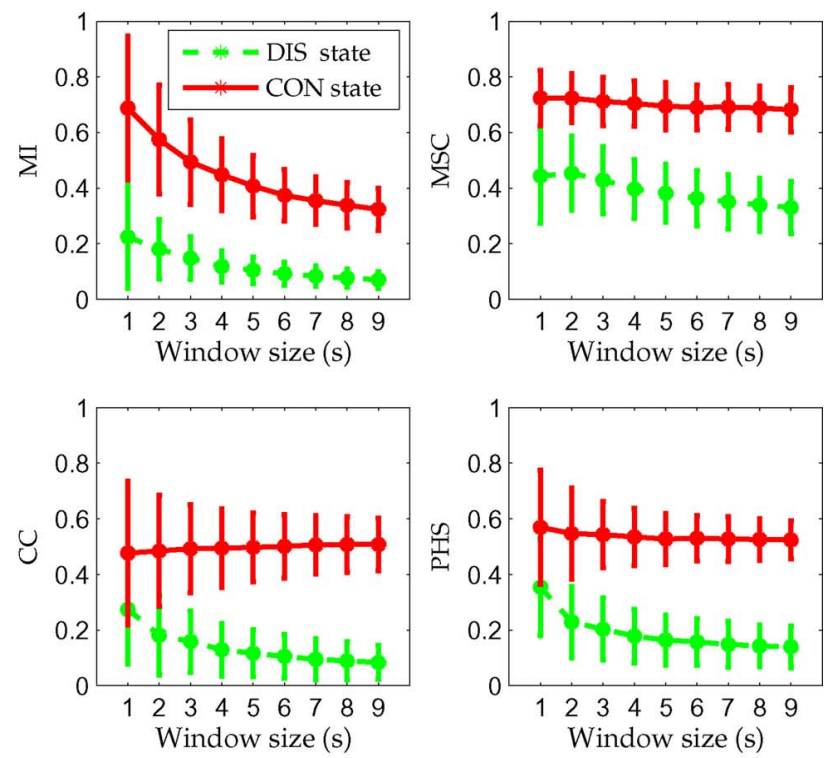

(b)

Fig. 3. Small sample size performance comparison among four dependence measures: MSC, CC, MI, and PhS, for detecting functional connectivity between spiking neuron A and B. (a) Statistical powers of the methods with respect to different estimation window sizes. We run the simulation model in 100 random settings. The error bar represents the standard derivation of the statistical power over 100 random settings. (b) The mean and standard deviation of each measure with respect to different estimation window sizes.

TABLE I

Statistical Powers of the Methods With Respect to DifFERENT Average Synaptic Weight W, When the Window Size IS 10 SAmples

\begin{tabular}{|c||c|c|c|c|}
\hline Method Weight & 1 & 1.5 & 2 & 2.5 \\
\hline \hline MSC & 0.34 & 0.40 & 0.64 & 0.77 \\
CC & 0.17 & 0.17 & 0.37 & 0.51 \\
MI & 0.44 & 0.64 & 0.83 & 0.84 \\
PhS & 0.18 & 0.38 & 0.38 & 0.64 \\
\hline
\end{tabular}

should compare the methods in the small window size case. The standard deviation of MSC ( 0.10 for CON state and 0.18 for DIS state) is the smallest when window size is 10 samples, while the standard derivation of MI $(0.25$ for CON state and 0.18 for DIS state) is relatively larger, which implies that MSC is able to estimate the strength of the functional connection more precisely.

In summary, the comparison reveals that MSC is a relatively reliable estimator of functional connectivity with short time resolution and will be our choice in this work.

\section{Cortical Neural Data}

The data for these experiments was collected in Nicolelis' primate laboratory at Duke University (see [32] for details). For our experiments, neural data are recorded from an owl monkey's cortex when the animal is performing a food reaching task. Multiple micro-wire arrays are used to record this data from 104 neural cells in the following four cortical areas: posterior parietal cortex $(P P-$ contra $)$, primary motor cortex (MI-contra), dorsal premotor cortex (PMD-contra), and primary motor and dorsal premotor (M1/PMD-ipsi). In tandem with the neural data recording, the 3-D hand positions are digitized when the monkey is performing the task.

For dependence analysis, neuronal spike events are binned in nonoverlapping windows of $100 \mathrm{~ms}$. The hand position data sets are digitally low-pass-filtered to avoid aliasing and downsampled to $10 \mathrm{~Hz}$ to match the binning utilized in the neural data. Taking the primate's reaction time into account, the spike trains were delayed by $0.230 \mathrm{~s}$ with respect to the hand position [33]. Our particular data set contains 104 neural channels recorded for $38.33 \mathrm{~min}$. This time recording corresponds to a data set of $23000 \times 104$ time bins, for which about 250 reaches are performed. The time spent in movement and resting are around 11 and 27 min, respectively.

\section{KinEMatic State AnALYsis}

Our testing with real data will elucidate the adequacy of the dependence measures to quantify and analyze neural data given the knowledge of the kinematic variables. Conditioning in the kinematic variables is just a way to segment the data for our statistical tests and does not invalidate the assumption that only neural data interactions are being utilized in the analysis. If the results with this segmentation show statistical different values for the different phases of the movements, then we have demonstrated that the dependence measures may be useful for motor control and other behavior experiments, even when no measured external variables are available. The only extra decision to be made by the experimenter in this more abstract framework is the selection of the baseline, the start of the analysis and the segment length. By brute force one can always use a small window and repeat the analysis in each and then perform clustering on the values obtained. For kinematic state analysis, our hypothesis is that specific neural assemblies are activated given a kinematic state and the goal of the analysis is to characterize assemblies by a set of neurons whose functional connectivity is enhanced significantly to define a state-related neural assembly. Specifically, the functional connectivity analysis of state-related assembly is based on a hypothesis test. Given a kinematic state, the pairwise neuron connections composing the state-related assembly are significantly higher than those in the rest state, which is against the null hypothesis that the connections between a pair 

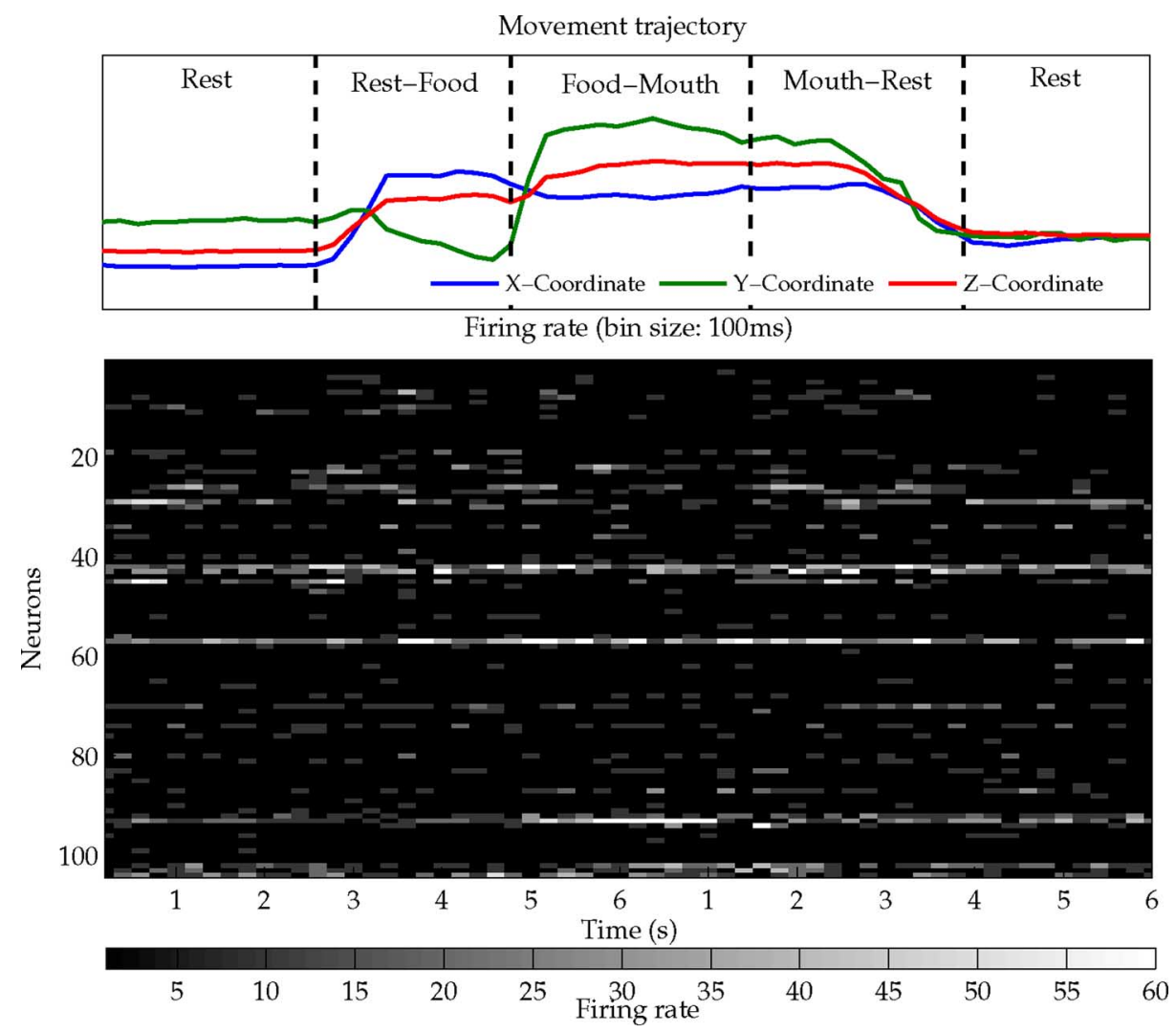

Fig. 4. Example of reaching movement trajectory. Trajectory is segmented into rest (R), rest-to-food (Mv1), food-to-mouth (Mv2), and mouth-to-rest (Mv3) states.

of neurons share the same distribution in the rest state and the given kinematic state. Moreover, a graph based on those pairwise functional connections are built to describe a state-related assembly. In the graph, the nodes and the edges represent the index of neurons and the functional connectivity between a pair of neurons.

\section{A. State-Related Assembly}

For the experiments, the neural data are segmented into the rest state and three movement states: rest-to-food (Mv1), food-to-mouth (Mv2), mouth-to-rest (Mv3) according to the previous segmentation of the 3-D hand trajectory [15], as shown in Fig. 4. The MSC-based measure is applied to estimate the functional connectivity of pairwise neurons in each segment. Since the average duration of each movement state is only $1 \mathrm{~s}$, the estimation window size is selected as $1 \mathrm{~s}$ (10 samples), and we further assume that the interaction within the state-related assembly during each state remains stationary. MSC between pairwise neurons is estimated for 50 trials belonging to each movement state and 100 trials during rest state, which is sufficient to determine the state-related assembly. In future work, testing whether these assemblies were in fact stable across the reaches will be conducted by comparing the assemblies estimated from different trail subsets.

The estimated dependence for each trial is treated as an observation of the conditional probability distribution given a kinematic state. We perform a KS-test, which gives an indication of the separability of two measurement sets, to check whether the pairwise neuron dependence in a movement state and the rest state are significantly different. The null hypothesis is that the connection between a pair of neurons share the same distribution in the rest state and the given movement state, which is translated for the KS test on nonsignificant difference of measured functional connection between these two states. Therefore, a rejection of null hypothesis at the 0.0001 significance level indicates that the connection between a pair of neurons is significantly different during the given state. The small significance level 0.0001 is determined by Bonferroni's correction for multiple comparisons where we divide a family wise error rate of 0.05 by the number of comparisons. Given a movement state, all activated connections (edges) and the connected neurons (nodes) comprise the assembly graph. The degree of a neuron is defined as the number of edges incoming to the neuron. Three conditional assembly graphs given three movement states are plotted based on KS-test results in Fig. 5.

For each figure, the black dots denote the activated functional connections (edges) at the movement state, compared to the rest state. In fact, in any of the movement states the dependence is always higher than in the rest state (figures not shown), which is consistent with the hypothesis that reciprocal interactions among neurons contribute to the emergence of the behavior [3]. What is more interesting is that the firing rate of neurons during resting and movement is not significantly different, as shown in Fig. 4.

The variability among these three graphs demonstrates the specificity of the state-related assemblies. In other words, the 

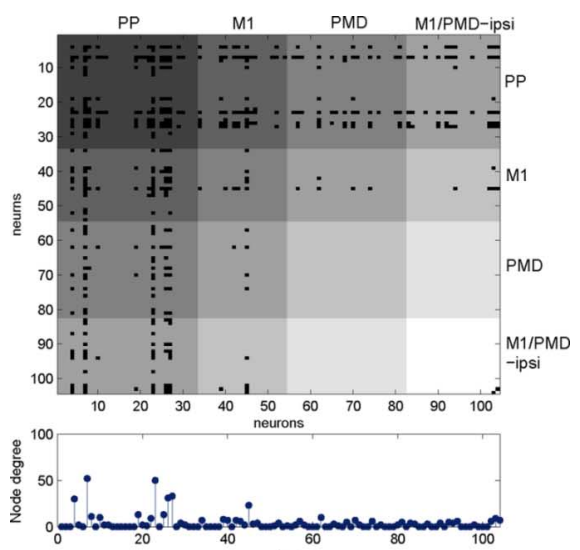

(a)
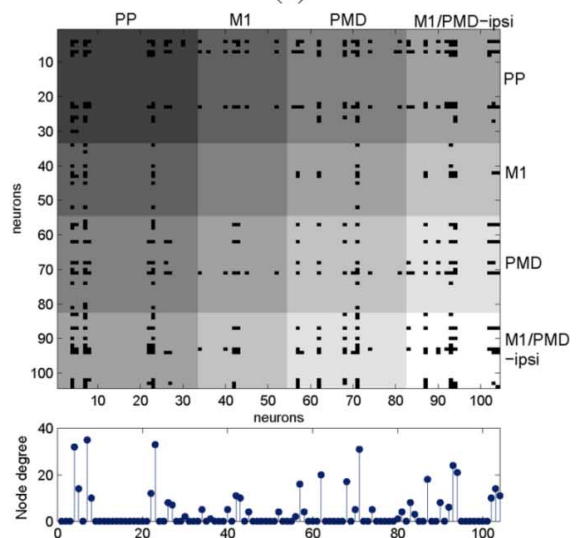

(b)
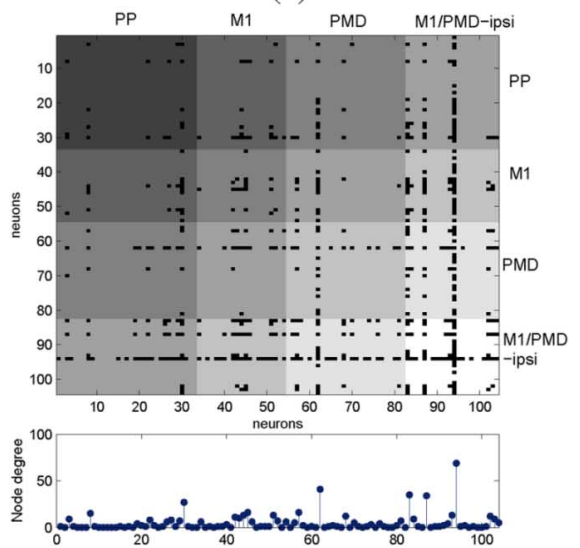

(c)

Fig. 5. $104 \times 104$ matrices plot three functional connectivity graphs assessed by KS-test results, which describe the state-related assembly in rest-to-food (Mv1), food-to-mouth (Mv2), and mouth-to-rest (Mv3) states. For each graph, the black dots denote the activated functional connections in this movement state with respect to the rest state. There are 104 neurons in the neural data set. Each point $(x, y)$ in the matrix represents the functional connection between neuron $x$ and neuron $y$. The background color of each graph is used to indicate the cortical area. The bottom plots in each sub-figure show the neuron degree (the number of edges incoming to the neuron) in the assembly graph given a movement state. (a) Rest-to-food (Mv1) state. (b) Food-to-mouth (Mv2) state. (c) Mouth-to-rest (Mv3) state.

state-related assemblies provide a subset of the cortical neurons that are especially important to emergence and implementation of a specific behavior. For example, in the initiation of the Mv2 movement there is functional connectivity among posterior parietal neurons. This connectivity spreads to multiple cortices (PMD-contra and M1-contra) during the large excursion reach between the food and mouth. To further investigate the correlation between the functional connectivity in state-related assemblies and behavioral states, we study the evolution of the connection strength and the activation degree of the state-related assemblies in the cortex.

\section{B. Evolution of Connection Strength}

The evolution of functional-connectivity strength is estimated by sliding a 1 or $3 \mathrm{~s}$ window by $0.1 \mathrm{~s}$ steps over the data. The results reveal that the strength of ensemble connections is enhanced at the movement states. To illustrate this connectivity evolution, the neurons connected to neuron 93 [see Fig. 5(c)] are selected from the assembly graph of the Mv3 assembly, which is one of the most connected neuron. The time-varying strengths of these connections are depicted in Fig. 6(a) and (b).

Fig. 6(a) demonstrates the dynamics of the connectivity associated with the Mv3 kinematic state with a $1 \mathrm{~s}$ moving window. The delay, the duration, and the peaks of the connection's strength vary among different neuron pairs. However, the connectivity pattern repeats at every reaching task, with the variance induced by slight movement differences across trials. These results support the hypothesis that the connection strength in state-related assembly based on KS-test is modulated by the kinematic state.

In Fig. 6(a), it is worth noting that MI and MSC are able to detect the association between the functional connectivity and the kinematic state with a fine time resolution. In contrast, cross-correlation and phase synchronization fail to detect such association with the $1 \mathrm{~s}$ estimation window. When the window increases to $3 \mathrm{~s}$ [Fig. 6(b)], the connection strength based on phase synchronization and cross correlation also shows the association with the transition from rest to movement states, but with relatively lower level and worse time resolution. The comparison further demonstrates the robustness of MSC and MI in detecting the functional connectivity with short estimation window.

\section{Activation Degree of State-Related Assemblies}

So far we have focused on individual neurons, now we move focus to neural assemblies. It is nontrivial to extend the pairwise dependencies to a measure pertaining to the total assembly activity. Hence, we are interested in how to quantify the degree of activation of an assembly by combining the estimated pairwise functional connections reported above. To compare the validity of the qualifications, we investigate the correlation between the activation degree of state-related assemblies and the specific kinematic states.

Specifically, the threshold for functional connection between each pair of neurons is estimated from the rest state set of pairwise dependence with a p-value of 0.05 . If the dependence of a pair of neurons is over the threshold, the corresponding edge (linking the node pair) and nodes in the assembly graph are activated. There are two possible ways to define the degree of the assembly activation: 1) the probability of the activated edge (edge method) defined as

$$
p_{\text {edge }}=\frac{n_{\text {edge }}}{N_{\text {edge }}} .
$$




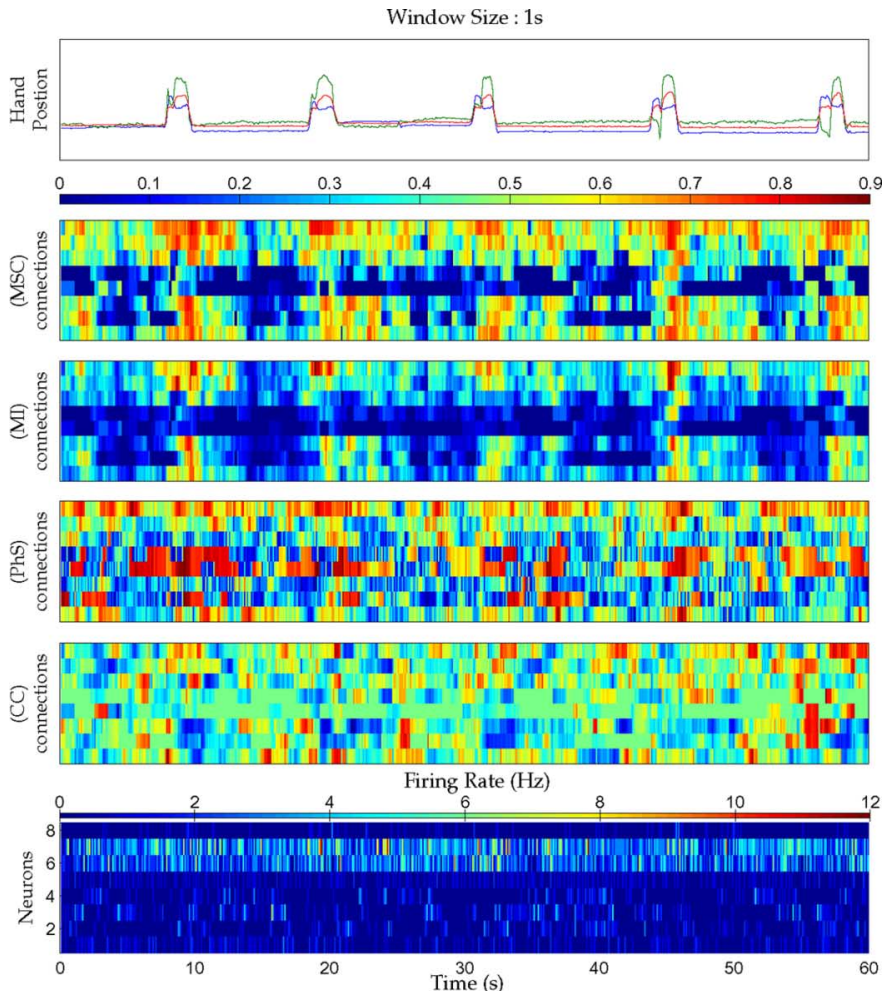

(a)
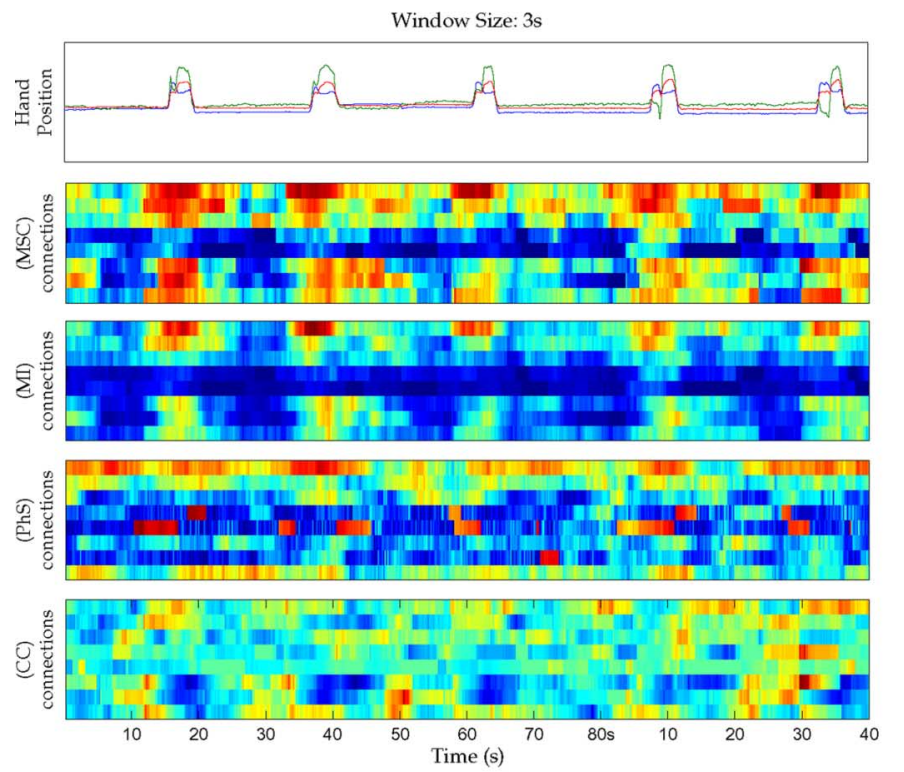

(b)

Fig. 6. Time-varying connection strength of neuron 93 in the Mv3 assembly. In each column sub-figure, the first panel shows the 3-D hand position time series, followed by the connection strength estimated by MSC, MI, PhS, and CC with a 1 or $3 \mathrm{~s}$ moving windows (sliding by $0.1 \mathrm{~s}$ steps). The color represents the connection strength of neuron 93 to its functionally connected neurons. The bottom plot in Fig. 6(a) shows the firing rates of neurons 93 and its functional connected neurons. (a) Window size: $1 \mathrm{~s}$. (b) Window size: $3 \mathrm{~s}$.

2) The probability of the activated nodes in the assembly graph (node method) defined as

$$
p_{\text {node }}=\frac{n_{\text {node }}}{N_{\text {node }}}
$$

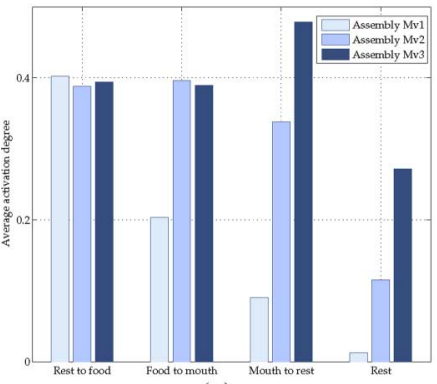

(a)

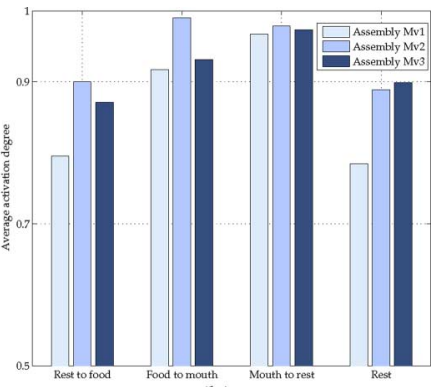

(b)
Fig. 7. Average activation degree of state-related assemblies. (a) Average activation degree of the assembly based on the activated edge. (b) Average activation degree of the assembly based on the activated node.

where $n_{\text {edge }}$ and $n_{\text {node }}$ represent the number of the activated edges and nodes and $N_{\text {edge }}$ and $N_{\text {node }}$ are the total number of the edges and nodes in the assembly graph. The edge-based activation degree measures the relative strength of the value of functional connections (interactions) in the given assembly. The node-based activation degree measures the relative number of neurons in the given assembly with enhanced functional connection.

For these three state-related assemblies, their average activation degree is estimated over all trails during Rest-to-food, food-to-mouth, mouth-to-rest, and rest kinematic states, respectively, as shown in Fig. 7. Fig. 7(a) shows the edge-based activation degree results, where given a state-related assembly, its activation degree always has the highest value during its corresponding kinematic state. From another aspect, during each movement state, its corresponding assembly always has the higher activation degree than the others. Moreover, the activation degrees of all three assemblies are decreased in the rest state. In contrast, there are no such results obtained by the node-based activation degree, as illustrated in Fig. 7(b).

In addition, the temporal evolution of functional activation estimated with the KS-test of three assemblies corresponding to Mv1, Mv2, and Mv3 using the edge and node methods are shown in Fig. 8. The assembly activation based on the edge method detects consistently the repeated food-reaching tasks. Moreover, the highest activation of the Mv1 assembly is only present in the beginning of the Mv1 state. The Mv2 assembly activation peaks during Mv2 and Mv3 state occurs. These results show that the activation of the assemblies is able to reach the peak repeatedly when the specific movement state arrives. These results also suggest that the specific assembly explains the emergence and operation of a specific behavior. In contrast, the assembly activation accessed by the node probability is less effective to detect the specific kinematic state because the same set of neurons is involved in different movements, in spite of the increased activation during the movement states. However, the organization of the interaction network appears more consistent given a specific kinematic state, and this is the reason why edges are better suited for discrimination of functional connectivity. This comparison reveals that the operation of a specific behavior is more closely associated with the network of interactions among the neurons. 

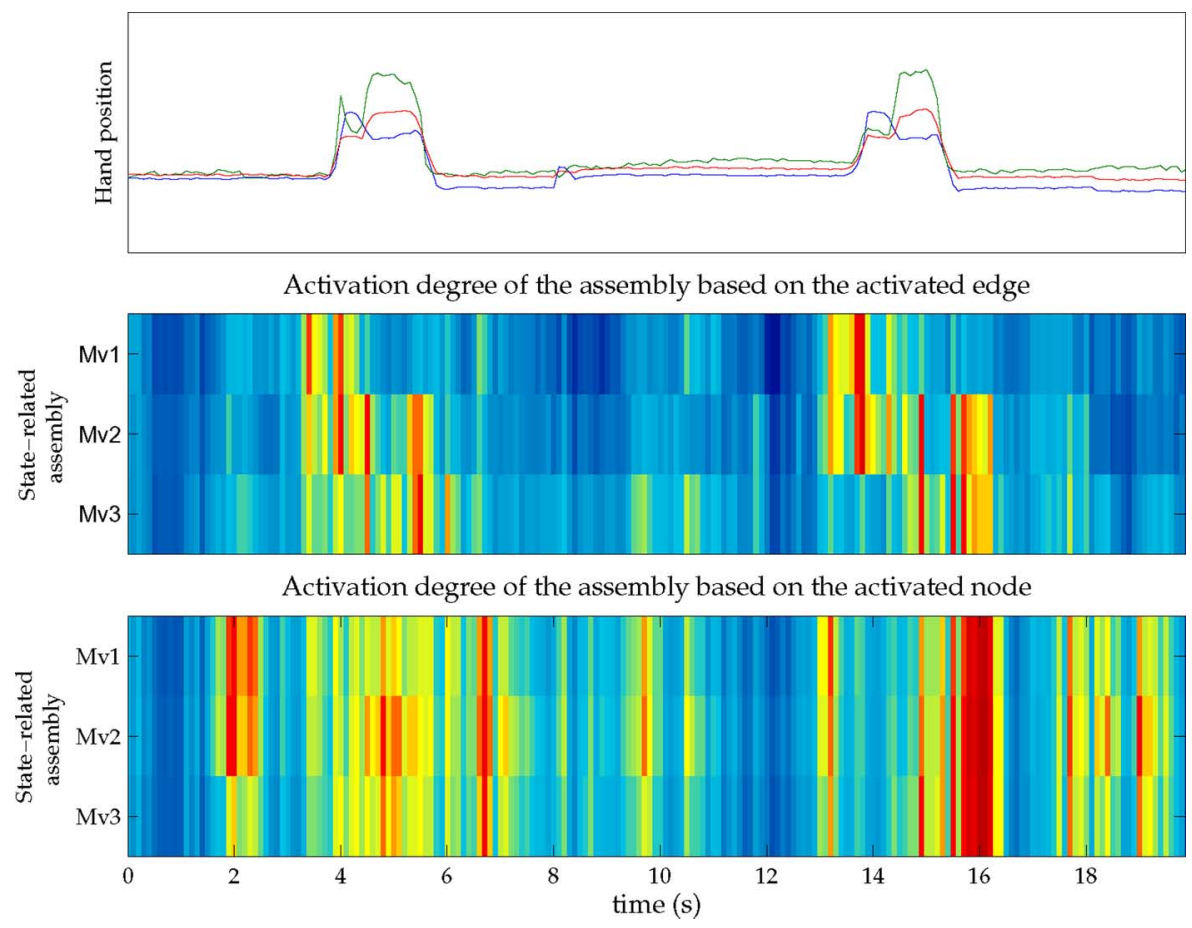

Fig. 8. The upper panel shows the reaching movement trajectory of 3-D hand position. The second panel is the activation degree of three neural assemblies based on the activated edge method, estimated by a moving window (sliding $1 \mathrm{~s}$ window by $0.1 \mathrm{~s}$ steps).The $y$-axis is the three assembly index corresponding the rest-to-food, food-to-mouth, and mouth-to-rest state. The third panel demonstrates the activation degree based on the activated node method.

\section{DYNAMICS OF LOCAL FUnCTIONAL CONNECTIVITY}

The different organization of the state-related assemblies (Fig. 5) motivates the question about the varying role of cortical regions in voluntary movement. To further investigate the relationship between cortical neural assemblies and behavior, the Flare toolkit [34] is applied to visualize the local assembly graphs of four cortical areas (PP-contra, M1-contra, PMD-contra, M1/PMD-ipsi).

In order to show the evolution of the graphs in time, we further separate the data into five substates, which are Mv1, Mv2, Mv3 and two middle states (the one between Mv1 and Mv2 and the one between Mv2 and Mv3), as shown in Fig. 9. The state-related assembly graphs are based on the KS-test results between each substate and the rest state, similarly to Section VII-A. In the interactive visualization of pairwise neural dependence graph created by Flare, the neurons are placed along a circle. A link indicates that two neurons are connected.

As shown in Fig. 9, the network in the PP-contra becomes highly interconnected before and at the beginning of the reaching task. It supports the role of PP-contra in producing planned movement and the linkage between internal to external coordinate systems.

In our results, the local functional connectivity within M1-contra is activated by the movement. Fig. 9(c) shows that when the velocity approaches zero in substate 3 , little interaction appears within this region (Fig. 9). Moreover, for the movements with different joint angles in substate 1 and substate 5 , most connections in assembly network are changed in M1-contra, which further supports the hypothesis that the specific assembly contributes to the emergence of the movement with specific kinematic parameters.
In addition, considerable functional connections are created during the mouth to rest state in M1/PMD-ipsi, but not during other kinematic states, which is consistent with the results of the hand trajectory reconstruction in [15]. They show that M1/PMD-ipsi accurately captures the mouth/rest regions, but misses the beginning of movement. Although the main organizational principle of primate motor systems is cortical control of contralateral limb movement, motor areas also appear to play a role in ipsilateral limb movements. Our results reveal that the interaction in M1 is modulated with functional connectivity by the ipsilateral limb movements, which support the argument that the motor areas are also able to correlate ipsilateral limb kinematics with high precision [35].

\section{CONCLUSION}

In this paper, we utilize dependence measures to quantify and study the dynamics of functional connectivity in motor cortex exclusively from the neural activity. Because the estimation window is limited by the time scale of kinematic states, the robustness of the functional-connectivity estimation with a small window size is key to detect the dynamic nature of neural activity. The comparison among four dependence measures demonstrates that MSC and MI are the most reliable estimators for one second windows which contain 10 samples of binned neural activity $(100 \mathrm{~ms})$. This bin size has been preferred for motor BMIs, but future studies should be conducted to verify that it is appropriate for functional studies as the one presented in this paper and if not, determine its optimal value. In addition, both MSC and MI are dependence measures that can be generalized to study multichannel data, so this extension will be investigated in our future work. 


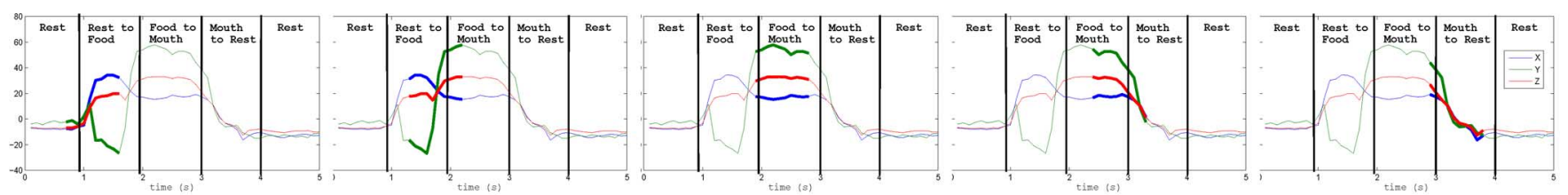

(a)
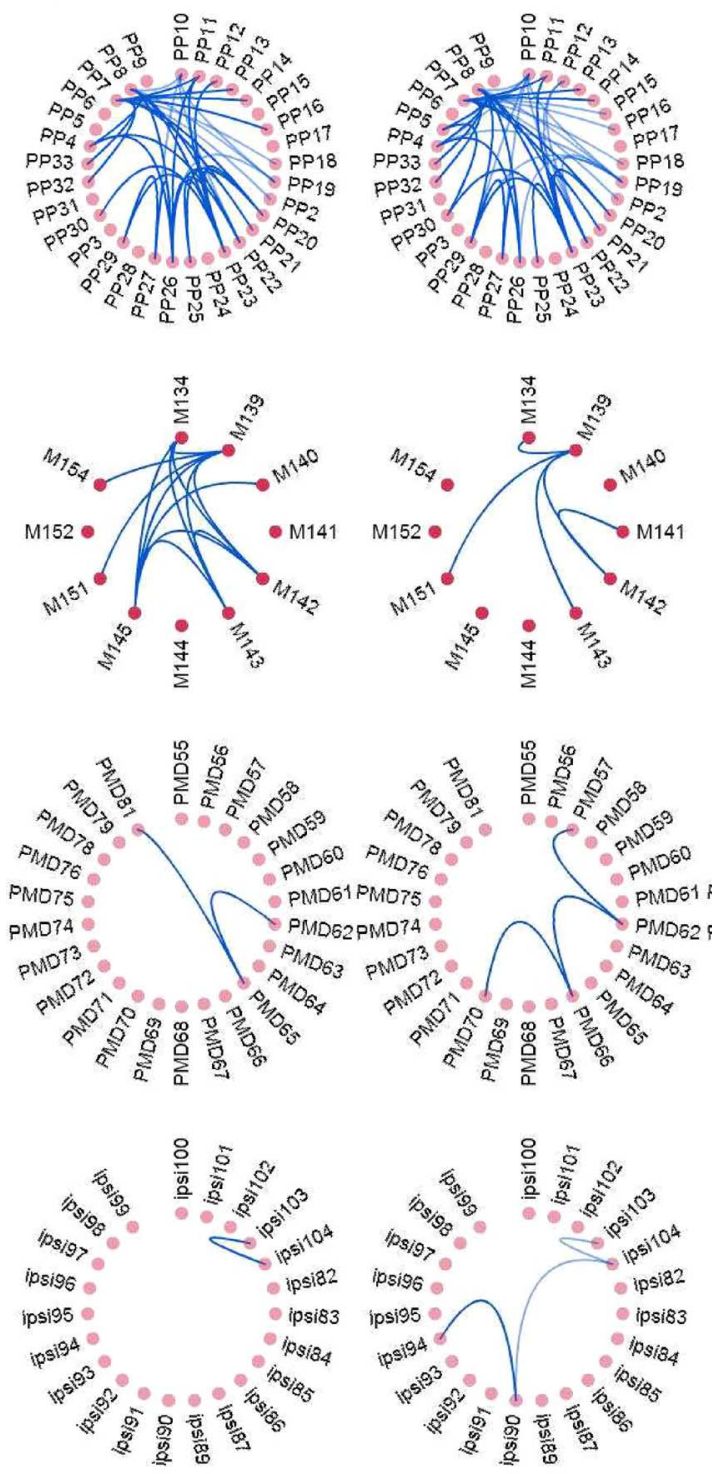

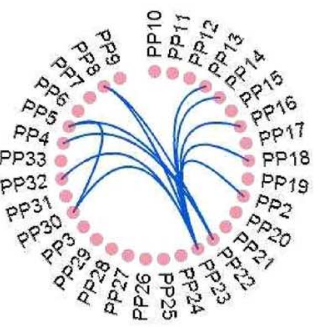

(b)

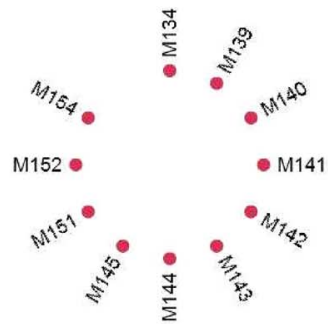

(c)

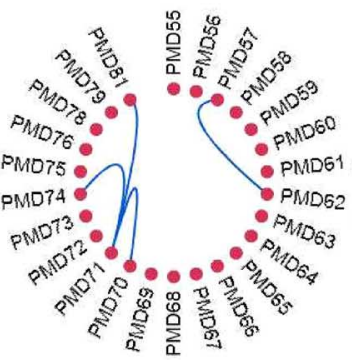

(d)

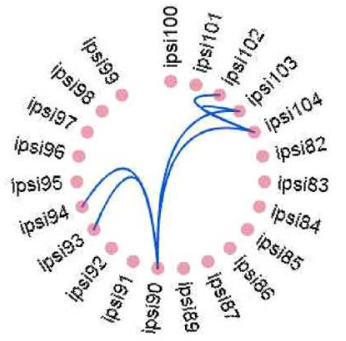

(e)
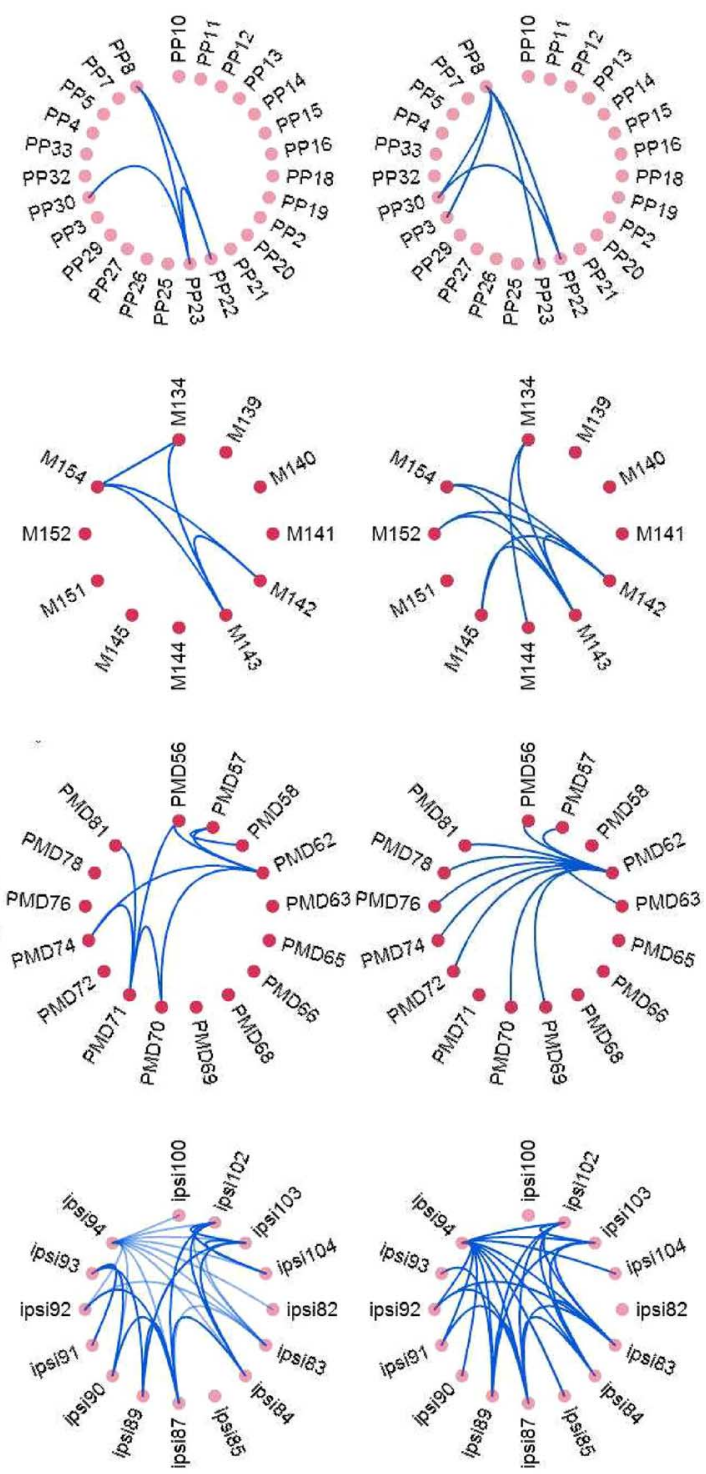

Fig. 9. Local functional connectivity graphs in four cortical areas: PP-contra, M1-contra, PMD-contra, and M1/PMD-ipsi for five movement substates. For each sub-figure, the five functional connectivity graphs correspond to the five movement substates. The neurons are paced along a circle and a link indicates that two neurons are connected. (a) Five substate of the reaching task trajectory. (b) Local functional connectivity graphs in PP-contra given the five movement substates. (c) Local functional connectivity graphs in M1-contra given the five movement substates. (d) Local functional connectivity graphs in PMD-contra given the five movement substates. (e) Local functional connectivity graphs in M1/PMD-ipsi given the five movement substates.

In this study, we test this algorithm with microelectrode array data for a food reaching task. Three state-related assemblies are assessed corresponding to the rest-to-food, food-to-mouth, and mouth-to-rest kinematic state, respectively. In spite of the coarse sampling of neural structures and the relatively coarse time resolution of the method, which imposed the grouping of all activated neurons in a single neural assembly, the graphs of conditional function connectivity show distinct assemblies.

Our approach detects a close association between the increase of functional connection strength in state-related assembly and the transition from rest to movement. Moreover, the activation degree of the state-related assembly repeatedly reaches the peak when the corresponding movement state occurs. In future work, the activation degree can be further investigated as a quantifiable way to estimate the specific movement state with functional connectivity. These results also reveal that the network of interactions among neurons seems the key factor for the operation of a specific behavior. Although in these experiments we conditioned on the kinematic variables, the method extracts the information solely from neural data, transcends motor tasks and 
can be applied to any brain area. In this more general scenario, the experimenter will have to select a baseline state, a starting point and a data length for statistical robustness (1 s currently), and apply the dependence measures for each window of neural data. Clustering of the dependency measures, or building the graphs to find consistency between dependencies across sequential segments can be used to abstract different interactions that are the signature for different kinematic states. The relationship to kinematics is merely used to support our conclusions. In future work, alternate statistical tests that are more specific than the KS test should be investigated.

The circle tree graphs of the local functional connectivity within each cortical area PP-contra, M1-contra, PMD-contra, and $M 1 / P M D$-ipsi help understand the time varying spatial assemblies that are in the cortex. Moreover, the dynamics of the local assembly graphs for each cortical area corroborate the regional contribution to the movement implementation. Since $P P$-contra localizes the body in space and plans the movement, neurons are highly interactive at the beginning of the reaching task. The interaction in M1-contra is only expressed when the movement velocity is large. These results also show that the functional connectivity is associated with ipsilateral limb movement. Although this was unexpected, we have shown by different analysis [15] that this only occurs during the latter portion of the trajectory.

In summary, the mean square contingency seems to be a valuable technique to inquire about functional activation of neural assemblies during behavior because of its statistical power at small sample size and its easy computation. One big challenge is how to shorten the observation window (now at least $1 \mathrm{~s}$ ) to increase the temporal resolution needed to study behavior. The other big challenge is how to fully utilize the information contained in the pairwise activations. Our approach was to apply a statistical significant threshold to simply the connectivity matrix and provide a first level analysis of functional connectivity. However, there is potentially much more information in the connection matrix to be quantified by for instance performing spectral clustering on this matrix to find and track clusters of functional activation over time. This will be pursued in further studies.

The dependence analysis of neuronal functional connectivity dynamics can provide an unsupervised estimation of the hidden neuronal states and their transitions related to the kinematic states. In addition, the state-related assembly also reveals the internal functional structure present in the multichannel of neural signals, which allows a more efficient utilization of the neural data in BMI applications, since neural modeling using multichannel data normally require an independence assumption amongst neural signals.

\section{REFERENCES}

[1] M. A. L. Nicolelis and M. A. Lebedev, "Principles of neural ensemble physiology underlying the operation of brain-machine interfaces," $\mathrm{Na}$ ture Rev. Neurosci., vol. 10, no. 7, pp. 530-540, Jul. 2009.

[2] M. Mesulam, "Large-scale neurocognitive networks and distributed processing for attention, language, and memory," Ann. Neurol., vol. 28, pp. 597-613, 2004.

[3] F. Varela, J. P. Lachaux, E. Rodriguez, and J. Martinerie, "The brainweb: Phase synchronization and large-scale integration," Nature Rev. Neurosci., vol. 2, pp. 229-239, 2001.
[4] A. M. Aertsen, G. L. Gerstein, M. K. Habib, and G. Palm, "Dynamics of neuronal firing correlation: Modulation of effective connectivity," $J$. Neurophysiol., vol. 61, no. 5, pp. 900-917, 1989.

[5] E. N. Brown, R. E. Kass, and P. P. Mitra, "Multiple neural spike train data analysis: State-ofthe-art and future challenges," Nature Neurosci., vol. 7, pp. 456-461, 2004.

[6] S. Grün, M. Diesmann, and A. Aertsen, "Unitary events in multiple single-neuron spiking activity: I. Detection and significance," Neural Computat., vol. 14, no. 1, pp. 43-80, Jan. 2002.

[7] G. Pipa, D. W. Wheeler, W. Singer, and D. Nikolić, "NeuroXidence: Reliable and efficient analysis of an excess or deficiency of joint-spike events," J. Computat. Neurosci., vol. 25, no. 1, pp. 64-88, Aug. 2008.

[8] F. Grammont and A. Riehle, "Spike synchronization and firing rate in a population of motor cortical neurons in relation to movement direction and reaction time," Biol. Cybern., vol. 88, pp. 360-373, 2003.

[9] A. S. Ecker, P. Berens, G. A. Keliris, M. Bethge, N. K. Logothetis, and A. S. Tolias, "Decorrelated neuronal firing in cortical microcircuits," Science, vol. 327, pp. 584-586, Jan. 2010.

[10] L. Li, S. Seth, I. Park, J. C. Sanchez, and J. Principe, "Neuronal functional connectivity dynamics in cortex: An MSC-based analysis," in IEEE Int. EMBS Conf., 2010.

[11] R. Q. Quiroga, A. Kraskov, T. Kreuz, and P. Grassberger, "Performance of different synchronization measures in real data: A case study on electroencephalographic signals," Phys. Rev. E, Stat. Phys. Plasmas Fluids Relat. Interdiscip. Top., vol. 65, no. 4, pp. 903-941, Mar. 2002.

[12] P. Berkes, F. Wood, and J. Pillow, "Characterizing neural dependencies with copula models," Adv. Neural Inf. Process. Syst., vol. 21, pp. 129-136, 2009

[13] Csim: A Neural Circuit Simulator The IGI LSM Group, 2006 [Online]. Available: http://www.lsm.tugraz.at

[14] M. Aghagolzadeh, S. Eldawlatly, and K. Oweiss, "Synergistic coding by cortical neural ensembles," IEEE Trans. Inf. Theory, vol. 56, no. 2, pp. 875-889, Feb. 2010.

[15] J. C. Sanchez, "From cortical neural spike trains to behavior: Modeling and analysis," Ph.D. dissertation, Univ. Florida, Gainesville, 2004.

[16] S. Darmanjian, "Design and analysis of generative models for brain machine interfaces," Ph.D. dissertation, Univ. Florida, Gainesville, 2009.

[17] J. Sanchez and J. C. Principe, Brain Machine Interface Engineering. San Rafael, CA: Morgan Claypool, 2007.

[18] S. Eldawlatly, R. Jin, and K. G. Oweiss, "Identifying functional connectivity in large-scale neural ensemble recordings: A multiscale data mining approach," Neural Computat., vol. 21, pp. 450-477, 2009.

[19] K. Pearson, J. Harris, A. Treloar, and M. Wilder, "On the theory of contingency," Am. Stat. Assoc., vol. 25, no. 171, pp. 320-327, Sep. 1930.

[20] R. Sokal and F. Rohlf, Biometry: The Principles and Practice of Statistics in Biological Research, 3rd ed. New York: Freeman, 1995.

[21] S. Yamada, K. Matsumoto, M. Nakashima, and S. Shiono, "Information theoretic analysis of action potential trains II. Analysis of correlation among yt neurons to deduce connection structure," J. Neurosci. Methods, vol. 66, pp. 35-45, 1996.

[22] M. S. Roulston, "Estimating the errors on measured entropy and mutual information," Physica D: Nonlinear Phenomena, vol. 125, pp. 285-294, 1999.

[23] E. Pereda, R. Q. Quiroga, and J. Bhattacharya, "Nonlinear multivariate analysis of neurophysiological signals," Progress Neurobiol., vol. 77, pp. 1-37, 2005

[24] S. Mallat, A Wavelet Tour of Signal Processing, Third ed. New York: Academic, 2008, The Sparse Way.

[25] M. Rosenblum, A. Pikovsky, J. Kurths, C. Schafer, and P. A. Tass, Phase Synchronization: From Theory to Data Analysis. New York: Elsevier, 2001, ch. 9, pp. 279-321.

[26] J. P. Lachaux, E. Rodriguez, J. Martinerie, and F. J. Varela, "Measuring phase synchrony in brain signals," Human Brain Mapp., vol. 8, pp. 194-208, 1999.

[27] P. Tass, M. G. Rosenblum, J. Weule, J. Kurths, A. Pikovsky, J. Volkmann, A. Schnitzler, and H. J. Freund, "Detection of $n: m$ phase locking from noisy data: Application to magnetoencephalography," Phys. Rev. Lett., vol. 81, no. 15, pp. 3291-3294, Oct. 1998.

[28] F. Mormann, K. Lehnertz, P. David, and C. E. Elger, "Mean phase coherence as a measure for phase synchronization and its application to the EEG of epilepsy patients," Physica D: Nonlinear Phenomena, vol. 144, no. 3-4, pp. 358-369, 2000.

[29] Y. Roudi, S. Nirenberg, and P. E. Latham, "Pairwise maximum entropy models for studying large biological systems: When they can work and when they can't,' PLoS Comput. Biol., vol. 5, no. 5, May 2009. 
[30] W. Maass, T. Natschläger, and H. Markram, "Real-time computing without stable states: A new framework for neural computation based on perturbations," Neural Computat., vol. 14, pp. 2531-2560, 2002.

[31] J. H. Zar, Biostatistical Analysis, T. Ryu, Ed. Englewood Cliffs, NJ: Prentice Hall, 1999.

[32] M. A. L. Nicolelis, "Brain-machine interfaces to restore motor function and probe neural circuits," Nature Rev. Neurosci., vol. 4, pp. 417-422, 2003.

[33] Y. Wang, "Point process Monte Carlo filtering for brain machine interface," Ph.D. dissertation, Univ. Florida, Gainesville, 2008.

[34] UC Berkeley Visualization Lab, 2009 [Online]. Available: http://flare. prefuse.org/

[35] K. Ganguly, L. Secundo, G. Ranade, A. Orsborn, E. F. Chang, D. F. Dimitrov, J. D. Wallis, N. M. Barbaro, R. T. Knight, and J. M. Carmena, "Cortical representation of ipsilateral arm movements in monkey and man,” J. Neurosci., vol. 29, pp. 12948-12956, 2009.

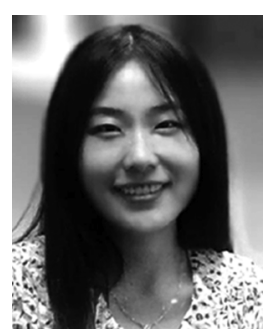

Lin $\mathbf{L i}\left(S^{\prime} 10\right)$ received the B.E. degree in electrical and information engineering from University of Science and Technology, Beijing, China, in 2007, the M.E. degree in electrical and computer engineering, in 2010, from University of Florida, Gainesville, where she is currently pursuing the Ph.D. degree in electrical and computer engineering.

She has been working in the Computational NeuroEnginnering laboratory at the University of Florida under the supervision of Dr. J. C. Principe since 2009. Her research interests are machine learning, adaptive signal processing, and computational neuroscience.

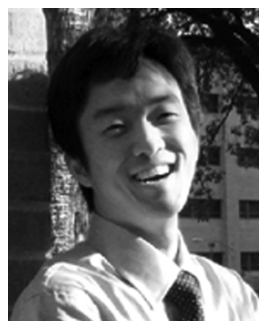

Il (Memming) Park was born in Germany, in 1979. $\mathrm{He}$ received the B.S. degree in computer science from KAIST, Daejeon, South Korea, in 2005, and the M.S. degree in electrical engineering and the $\mathrm{Ph} . D$. degree in biomedical engineering from the University of Florida, Gainesville, in 2007 and 2010, respectively.

He is currently a post-doc at the Center for Perceptual Systems, and Institute for Neuroscience at University of Texas at Austin. His research interests include statistical modeling and analysis of spike trains, information theoretic learning, Bayesian inference, and computational neuroscience.

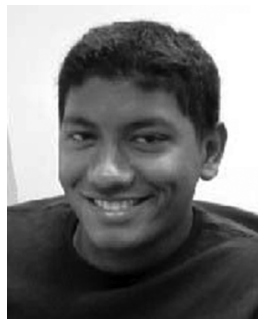

Sohan Seth (M'11) received the Ph.D. degree from the Electrical and Computer Engineering Department, the University of Florida, Gainesville, in 2011.

Currently he is a post-doctoral researcher at the Helsinki Institute for Information Technology, Helsinki, Finland. His research interests lie in the fields of machine learning, data mining, and computational neuroscience.

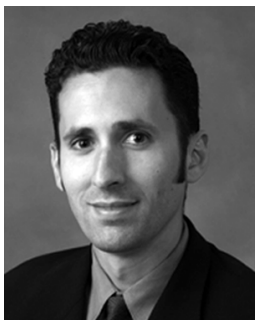

Justin C. Sanchez (M'02) received the B.S. degree in engineering science (with highest honors) with a minor in biomechanics, in 2000, and the M.E. and $\mathrm{Ph} . \mathrm{D}$. degrees in biomedical engineering from the University of Florida, Gainesville, in 2004.

$\mathrm{He}$ is the Director of the Neuroprosthetics Research Group (NRG). As an Associate Professor of Biomedical Engineering, Neuroscience, and a faculty member of the Miami Project to Cure Paralysis at the University of Miami, his mission is to develop state-of-the-art neural interface medical treatments. His laboratory is innovating neurotechnology for treating, paralysis, stroke, DBS for movement disorders, Tourettes, and OCD. His neural engineering electrophysiology laboratory has published over 75 peer review papers, seven patents, and one book in neuroprosthetic design.

Dr. Sanchez is an elected member of the Administrative Committee of the IEEE Engineering in Medicine and Biology Society. He is a reviewer for the NIH, DOD, and Associate Editor/reviewer of multiple journals of biomedical engineering.

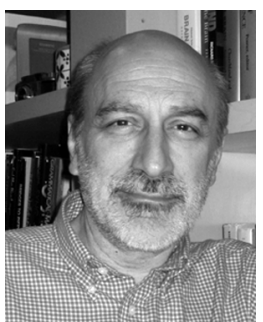

José C. Príncipe (F'00) is a Distinguished Professor of Electrical and Computer Engineering with the University of Florida, Gainesville. He is a BellSouth Professor and the Founding Director of the Computational Neuro-Engineering Laboratory, University of Florida. His current research interests are centered in advanced signal processing and machine learning, brain-machine interfaces, and the modeling and applications of cognitive systems. 